\title{
On Discretizations of the Generalized Boole Type Transformations and their Ergodicity
}

Prykarpatski AK*

Department of Applied Mathematics, AGH University of Science and Technology, Poland

\begin{abstract}
There is studied an analytical discretization of the generalized Boole type transformations in Rn and their ergodicity properties. The fixed points of the corresponding finite-dimensional stochastic Frobenius-Perron operator discretization are constructed, the structure of the related invariant measures is analyzed.
\end{abstract}

Keywords: Frobenius-Perron operator; Discretizatrion; Invariant measure; Ergodic measure; Boole type transformations; Ergodicity and mixing

\section{Introduction: The Frobenius-Perron Operator and Its Discretization}

We consider an $m$-dimensional; not necessary compact; $C^{1}$ manifold $M^{m}$, endowed with a Lebesgue measure $\mu$ determined on the $\sigma$-algebra of Borel subsets of $M^{m}$ and $\phi: M^{m} \rightarrow M^{m}$ being an almost everywhere smooth mapping. The related [1-5] Frobenius-Perron operator

$$
\mathcal{P}_{\varphi}: L_{1, l o c}\left(M^{n} ; \mathbb{R}\right) \rightarrow L_{1 . l o c}\left(M^{n} ; \mathbb{R}\right)
$$

is defined by means of the integral relationship

$$
\int_{A} \mathcal{P}_{\varphi} h d \mu:=\int_{\varphi^{-1}(A)} h d \mu
$$

for any $h \in L_{1, l o c}\left(M^{n} ; \mathbb{R}\right)$ and all $\mu$-measurable subsets $A \subset M^{m}$ Equivalently it can be defined as a mapping on the measure space $\mathcal{M}\left(M^{m}\right)$

$$
\mathcal{P}_{\varphi} \boxplus v \otimes(A):=v\left(\varphi^{-1}(A)\right)
$$

for any measure $v \in \mathcal{M}\left(M^{m}\right)$ and all $\mu$-measurable subsets $A \subset M^{m}$ In particular; if a measure $v \in\left(M^{m}\right)$ is absolutely continuous with respect to the measure $\mu$ on $M^{m}$ then definitions (3) and (2) are equivalent. In the infinitesimal form the Frobenius-Perron operator (1) action is representable as

$$
\mathcal{P}_{\varphi} h(x)=\sum_{y(x) \in \varphi^{-1}(x)} h(y(x))\left|\frac{d \mu(y(x))}{d \mu(x)}\right|=\sum_{y(x) \in \varphi^{-1}(x)} h(y(x))\left|\frac{d \mu(\varphi(y))}{d \mu(y)}\right|_{y=y(x)}^{-1}
$$

for any $B_{i} \subset M^{m}, i=\overline{1, N}$, and $x \in M^{m}$, where $d \mu(\phi(y)) / d \mu(y)$ means the usual Radon-Nikodym derivative $[1,3]$ of the shifted measure $\mu . \phi$ with respect to the Lebesgue measure $\mu$ on $M^{m}$ As we are mainly interested in studying the ergodic properties of the mapping $\phi: M^{m} \rightarrow M^{m}$ by means of the finite dimensional tools; we now proceed to a discretization approach [6-8] to the Frobenius-Perron operator (1) preliminarily choosing a partition $\mathcal{B}_{N}$ of the manifold $M^{m}$ as $N \in Z_{+}$boxes (or sells) $B_{i} \subset M^{m}, i=\overline{1, N}$, and introducing the space $\mathcal{L}^{N}$ of the step-functions on $M^{m}$ with respect to the partition $\mathcal{B}_{N}$ which can be constructed using the projection operator $\Pi_{N}: L_{1, l o c}\left(M^{n} ; \mathbb{R}\right) \rightarrow \mathcal{L}^{N} \subset L_{1, l o c}\left(M^{n} ; \mathbb{R}\right):$

$$
\left(\Pi_{N} h\right)(x):=\frac{\chi_{B_{i}}(x)}{\mu\left(B_{i}\right)} \int_{B_{i}} h d \mu
$$

for any $h \in L_{1, l o c}\left(M^{n} ; \mathbb{R}\right)$ and all $x \in M^{m}$ Then; by definition; one can define the Frobenius-Perron operator discretization as

$$
\mathcal{P}_{\varphi, N}:=\left.\Pi_{N} \mathcal{P}_{\varphi}\right|_{\mathcal{L}^{N}} .
$$

As a consequence of the definitions above one obtains that the discretized Frobenius-Perron operator (6) can be represented with respect to the canonical basis in the finite-dimensional space $\mathcal{L}^{N}$ by means of the $(N \times N)$ matrix

$$
\mathcal{P}_{\varphi, N}=\left\{\mathcal{P}_{\varphi, N}^{i j}:=\mu\left(\varphi^{-1}\left(B_{i}\right) \cap B_{j}\right) \mu\left(B_{j}\right)^{-1}: i, j=\overline{1, N}\right\},
$$

which is exactly a discretization of the infinitesimal expression (4). The matrix component $\mathcal{P}_{\varphi, N}^{i j}, i, j=\overline{1, N}$, can be; obviously; interpreted as a transition probability matrix for a point in $B_{i}$, being randomly chosen with respect to the measure $\mu$ to be mapped into the set $B_{i}$ by the mapping $\phi: M^{m} \rightarrow M^{m}$. Thus; the obtained stochastic matrix $\mathcal{P}_{\varphi, N}: \mathcal{L}^{N} \rightarrow \mathcal{L}^{N}$ defines naturally a finite homogeneous Markov chain; and particularly a linear discrete dynamical system in the Euclidean space $\mathbb{E}^{N} \simeq \mathcal{L}^{N}$.

The described approach to study the dynamical properties of the mapping $\phi: M^{m} \rightarrow M^{m}$ by means of the discretized Frobenius-Perron operator (6) is widely used in the literature [6,8-11]. It was also effectively used S. Ulam for finding the approximation of the invariant measures for the mapping $\phi: M^{m} \rightarrow M^{m}$ which are related with nonnegative fixed points of the discretized Frobenius-Perron operator (6). In addition; the discretized Frobenius-Perron operator (6) appears to be very useful for analyzing the ergodicity and mixing properties

$[2,4,5,7,8]$ of the mapping $\phi: M^{m} \rightarrow M^{m}$. Namely; the ergodicity of it with respect to the partition $\mathcal{B}_{N}$ is defined as the irreducibility of the discretized Frobenius-Perron operator (6); and the mixing with respect to the partition $\mathcal{B}_{N}$ is defined as its primitivity and ergodicity.

\section{Discrete Ergodicity Analysis}

As ergodicity of the mapping $\phi: M^{m} \rightarrow M^{m}$ is deeply connected with the suitably determined ergodic measure $v$ on $M^{m}$, which is a special invariant measure on $M^{m}$ such that any $\phi$-quasi-invariant function $\phi: M^{m} \rightarrow M^{m}$ is almost everywhere constant on $M^{m}$ we will be mainly interested below in the invariant measure $v$ absolutely continuous with respect to the Lebesgue measure $\mu$ on $M^{m}$ which is a fixed point

*Corresponding author: Prykarpatski AK, Professor, Department of Applied Mathematics, AGH University of Science and Technology, Poland, Tel: +48 12617 22 22; E-mail: pryk.anat@ua.fm, prykanat@cybergal.com

Received June 03, 2016; Accepted September 27, 2016; Published September 30, 2016

Citation: Prykarpatski AK (2016) On Discretizations of the Generalized Boole Type Transformations and their Ergodicity. J Phys Math 7: 199. doi: 10.4172/20900902.1000199

Copyright: ( 2016 Prykarpatski AK. This is an open-access article distributed under the terms of the Creative Commons Attribution License, which permits unrestricted use, distribution, and reproduction in any medium, provided the original author and source are credited. 
of the Frobenius-Perron operator (1); defined by the mapping (3). In what follows there is accepted the next [12] definition of the discrete ergodicity.

Definition 2.1: A measurable mapping $\phi: M^{m} \rightarrow M^{m}$ is called ergodic with respect to the partition $\mathcal{B}_{N}$ if the following discrete ergodic theorem holds:

there exists a non-negative definite and normalized vector $H^{(0)} \in \mathbb{E}^{N}, H^{(0)} \geq 0,\left\|H^{(0)}\right\|_{1}=1$, such that

$$
\lim _{n \rightarrow \infty} \frac{1}{n} \sum_{k=0}^{n-1} \mathcal{P}_{\varphi, N}^{k} H=H^{(0)}
$$

for any $H \in \mathbb{E}^{N}, H \geq 0,\|H\|_{1}=1$.

It is naturally to assume that the discrete ergodicity with respect to the partition $\mathcal{B}_{N}$ can happen to be persisting for almost all possible partitions of $M^{m}$ and for arbitrary dimensions $N \in \mathbb{Z}$. In this case one can determine a set of functions $\left\{h_{N}^{(0)}: M^{m} \rightarrow \mathbb{R}_{+}: N \in \mathbb{Z}_{+}\right\}$, where

$$
h_{N}^{(0)}(x):=\sum_{j=1}^{N} H_{j}^{(0)} \chi_{B_{j}}(x)
$$

for any $x \in M^{m}$ and next proceed to studying the existence of the pointwise limiting function

$$
h^{(0)}(x):=\lim _{N \rightarrow \infty} h_{N}^{(0)}(x)
$$

defining the corresponding absolutely continuous with respect to the measure $\mu$ on $M^{m}$ and invariant with respect to the transformation $\phi: M^{m} \rightarrow M^{m}$ measure

$$
v(A):=\int_{A} h^{(0)} d \mu
$$

for any measurable subset $A \subset M^{m}$. If the constructed measure (11) proves to be finite; that is $\int_{M^{m}} h^{(0)} d \mu<\infty$, then this invariant measure $v$ can be easily made probabilistic.

Taking into account the fact that the Frobenius-Perron matrix (7) is stochastic; one can recall the well known Frobenius-Perron theory [13] of non-negative stochastic matrices; in particular the following useful proposition.

Proposition 2.2: The mapping $\phi: M^{m} \rightarrow M^{m}$ is with respect to the partition $\mathcal{B}_{N}$ :

ergodic iff the matrix $\mathcal{P}_{\phi N}$ is irreducible; that is for every pair of states $(i, j)$ it is possible to move from $i$ to $j$ and back again; in other words $\mathcal{P}_{\phi, N}$ is irreducible; if it is not block upper-triangular; up to reordering rows and columns;

mixing iff the matrix $\mathcal{P}_{\phi, N}$ is primitive; that is all its eigenvalues not equal to the unity have modulus less than unity;

ergodic; but not mixing; iff the matrix $\mathcal{P}_{\phi, N}$ is $q$-cycling with maximal $q>0$

Moreover; it is worthy of mentioning that the irreducibility and primitivity depend only on the structure of the directed graph $G_{N}$, naturally associated with the matrix $\mathcal{P}_{\phi, N}$ Concerning the effective studying of the sole ergodicity of the mapping $\phi_{: M}{ }^{m} \rightarrow_{M}{ }^{m}$ the following famous Frobenius-Perron theorem proves strongly important.

Proposition 2.3: An irreducible stochastic matrix $\mathcal{P}_{\phi, N}$ is -cyclic with $q \in Z_{+}$maximal iff one of the following equivalent conditions holds:

a) There are $q$ different eigenvalues of the matrix $\mathcal{P}_{\phi, N}$ of modulus one;

b) There are $q$ symmetrically distributed and algebraically simple eigenvalues $\exp (2 \pi i k / q), k=\overline{0, q-1}$, of the matrix $\mathcal{P}_{\phi, N ;}$ c) the whole spectrum of the matrix $\mathcal{P}_{\phi, N}$ is invariant under the rotation about the angle $2 \pi / q$.

\section{The Classical Boole Mapping and Its Ergodicity}

The classical Boole transformation [14] $\phi: \mathbb{R} \rightarrow \mathbb{R}$ is defined as the almost everywhere smooth mapping

$$
\varphi(x):=x-1 / x,
$$

defined for all $x \in \mathbb{R} \backslash\{0\}$ It was proved to be ergodic [1,15] with respect to the standard invariant infinite Lebesgue measure on $\mathbb{R}$. The corresponding fixed point equation for the Frobenius-Perron operator action (4) can be easily presented as

$$
\left(\mathcal{P}_{\varphi} h^{(0)}\right)(x)=\sum_{ \pm} h^{(0)}\left(y_{ \pm}(x)\right) y_{ \pm}^{\prime}(x)=h^{(0)}(x),
$$

where; by construction; $\varphi\left(y_{ \pm}(x)\right)=x, \quad y_{ \pm}^{\prime}(x)>0$, and $h^{(0)}(x) \geq 0$ for almost all $x \in \mathbb{R}$ Having assumed that there exists an meromorphic continuation $h^{(0)}: \mathbb{C} \rightarrow \mathbb{C}$ of the mapping $h^{(0)}: \mathbb{R} \rightarrow \mathbb{R}_{+}$, such that $\left|h^{(0)}(z)-k^{(0)}\right|=O\left(1 /\left|z^{2}\right|\right)$ for $|z| \rightarrow \infty$ and some $k^{(0)} \geq 0$, the equality (13) can be rewritten as

$$
\begin{aligned}
& \sum_{ \pm}\left[h^{(0)}\left(y_{ \pm}(x)\right)-k^{(0)}\right] y_{ \pm}^{\prime}(x)= \\
& =-\lim _{r \rightarrow \infty} \frac{1}{2 \pi i} \frac{d}{d x} \sum_{ \pm \partial \mathbb{C}_{r}}\left[\ln \left(z-y_{ \pm}(x)\right]\left[h^{(0)}(z)-k^{(0)}\right] d z+\right. \\
& +\frac{1}{2 \pi i} \frac{d}{d x} \sum_{\{b=a, \bar{a}\}}\left[\ln _{O_{\varepsilon}(b\}}\left(z-y_{ \pm}(x)\right]\left[h^{(0)}(z)-k^{(0)}\right] d z=\right. \\
& =-\lim _{r \rightarrow \infty} \frac{1}{2 \pi i}{ }_{\partial O_{r}(0\}}\left[\frac{d}{d x} \ln \left(z^{2}-z x-1\right)\right]\left[h^{(0)}(z)-k^{(0)}\right] d z+ \\
& +\sum_{\{a\}_{\partial O} O_{\varepsilon}(a\}} \frac{z\left[h^{(0)}(z)-k^{(0)}\right] d z}{\left(z^{2}-z x-1\right)}+\sum_{\{\bar{a}\}_{\partial O_{\varepsilon}(\bar{a}\}}} \frac{z\left[h^{(0)}(z)-k^{(0)}\right] d z}{\left(z^{2}-z x-1\right)}= \\
& =\lim _{r \rightarrow \infty} \frac{1}{2 \pi i_{\partial O_{r}}(0)} \frac{z\left[h^{(0)}(z)-k^{(0)}\right] d z}{\left(z^{2}-z x-1\right)}+\left.\sum_{\{b=a, \bar{a}\}} \frac{k_{b}^{(0)}(x-2 z)}{\left(z^{2}-x z-1\right)^{2}}\right|_{z=b}= \\
& +\lim _{r \rightarrow \infty} \frac{1}{2 \pi i} i_{\partial O_{1 / r^{(0)}}} \frac{\left[h^{(0)}(1 / s)-k^{(0)}\right] d s}{s\left(1-x s-s^{2}\right)}+\left.\sum_{\{b=a, \bar{a}\}} \frac{k_{b}^{(0)}(x-2 z)}{\left(z^{2}-x z-1\right)^{2}}\right|_{z=b}= \\
& =\left.\sum_{\{b=a, \bar{a}\}} \frac{k_{b}^{(0)}(x-2 z)}{\left(z^{2}-x z-1\right)^{2}}\right|_{z=b},
\end{aligned}
$$

where $O_{r}(b):=\{|z-b|<r, b \in \mathbb{C}, \quad r>0\} \quad$ and $\quad k_{\bar{a}}^{(0)}=\bar{k}_{a}^{(0)}, \operatorname{Re}_{a}^{(0)} \geq 0, \quad$ are the corresponding residuum constants; related with the assumed finite second order pole set $\left\{a, \bar{a} \in \mathbb{C} \backslash \mathbb{R}\right.$ of the function $h^{(0)}: \mathbb{C} \rightarrow \mathbb{C}$, satisfying some finite system of algebraic constraints; ensuring the positivity of the reduced function $h^{(0)}: \mathbb{R} \rightarrow \mathbb{R}$. Based on simple enough yet cumbersome calculations one can get convinced that this system of constraints is compatible iff the constants $k_{b}^{(0)}=0$ for all $b \in\{a, \bar{a}\}$. Then from (5) one easily derives that

$$
\begin{gathered}
h^{(0)}(x)=k^{(0)} \sum_{ \pm} y_{ \pm}^{\prime}(x)=k^{(0)}\left[\frac{y_{+}(x)}{2 y_{+}(x)-x}+\frac{y_{-}(x)}{2 y_{-}(x)-x}\right]= \\
=k^{(0)} \frac{4 y_{+}(x) y_{-}(x)-x\left[y_{+}(x)+y_{-}(x)\right]}{4 y_{+}(x) y_{-}(x)+x^{2}-2 x\left[y_{+}(x)+y_{-}(x)\right]}=k^{(0)},
\end{gathered}
$$

where we made use of the obvious identities $y_{+}(x) y_{-}(x)=-1$ and 
$y_{+}(x)+y_{-}(x)=x$ for all $x \in \mathrm{R}$ Thus; the invariant infinitesimal measure with respect to the Boole mapping (12) equals

$$
d v(x)=k^{(0)} d x,
$$

being absolutely continuous subject to the standard Lebesgue measure $d x$ on R Thus; one can formulate the following theorem.

Theorem 3.1: Being unique; modulo the constant multiplier; the invariant with respect to the Boole mapping (12) measure expression (16) is ergodic on axis $\mathbb{R}$

Having now constructed the uniformly discretized FrobeniusPerron operator matrix (7); one can check that the matrix $\mathcal{P}_{\phi N}$ is reducible with respect to any partition $\mathcal{B}_{N}=\cup_{j=-N}^{N_{+}}[j / N,(j+1) / N] \subset \mathbb{R}$ for any its dimension $N:=\left(N_{-}+N_{+}\right) \rightarrow \infty$. Then; based on Proposition 2.2; one can claim that the Boole mapping (12) is ergodic with respect to any partition $\mathcal{B}_{N}, N \rightarrow \infty$ One can also verify that the positive definite vector $H^{(0)}=(1 / N, 1 / N, \ldots, 1 / N) \in \mathbb{E}^{N}$ solves the limiting condition (8); being its eigenvector for the unity eigenvalue:

$$
\mathcal{P}_{\varphi, N} H^{(0)}=H^{(0)}
$$

for any dimension $N \rightarrow \infty$ As a corollary of the claim above and the cycling properties of the Frobenius-perron matrix $\mathcal{P}_{\phi, N}$ one derives the next theorem; generalizing the one proved in [15] by means of different mostly qualitive tools.

Theorem 3.2: The Boole transformation (12) is ergodic; yet not mixing.

As it can be checked by means of direct computations; the Boole transformation (12) is ergodic yet not mixing; as the matrix $\mathcal{P}_{\phi, N}$ is $q_{N}{ }^{-}$ cycling with maximal $q_{N}>0$ for any dimension $N \rightarrow \infty$

\section{The generalized Boole Type Mapping and Its Ergodicity}

In the present section; we will study the invariant measures and ergodicity properties for the generalized Boole type transformations of plane $\mathbb{R}^{2}$

$$
\varphi_{1}\left(x_{1}, x_{2}\right):=\left(x_{1}-1 / x_{2}, x_{2}+1 / x_{1}\right), \quad \varphi_{2}\left(x_{1}, x_{2}\right):=\left(x_{1}+1 / x_{2}, x_{2}-1 / x_{1}\right),
$$

where $\left(x_{1}, x_{2}\right) \in \mathbb{R}^{2} \backslash\{(0,0)\}$. The corresponding to the mapping $\varphi_{1}: \mathbb{R}^{2} \rightarrow \mathbb{R}^{2}$ local Frobenius-Perron operator $\mathcal{P}_{\phi}$ acts on a non-negative definite function $h^{(0)}: \mathbb{R}^{2} \rightarrow \mathbb{R}_{+}$as

$$
\left(\mathcal{P}_{\varphi} h^{(0)}\right)\left(x_{1}, x_{2}\right)=\sum_{ \pm} h^{(0)}\left(y_{1, \pm}, y_{2, \pm}\right)\left[1+y_{1, \pm}^{-2} y_{2, \pm}^{-2}\right],
$$

where; by definition; $y_{1, \pm}:=y_{1, \pm}\left(x_{1}, x_{2}\right), y_{2, \pm}:=y_{2, \pm}\left(x_{1}, x_{2}\right), \varphi_{1}\left(y_{1, \pm}, y_{2, \pm}\right):=\left(x_{1}, x_{2}\right)$, $y_{1, \pm}^{2}-x_{1} y_{1, \pm}+x_{1} / x_{2}=0, \quad y_{2, \pm}=y_{1, \pm} x_{2} / x_{1}$ for any $\left(x_{1}, x_{2}\right) \in \mathbb{R}^{2} \backslash\{(0,0)\}$. It is easy to check by means of direct and simple enough calculations that a positive constant function $h^{(0)}\left(x_{1}, x_{2}\right)=k^{(0)} \in \mathbb{R}_{+}$is an eigenfunction of the mapping (19) with the unity eigenvalue:

$$
\mathcal{P}_{\varphi} k^{(0)}=k^{(0)} .
$$

This; in particular; means that the infinitesimal measure $\left.d v\left(x_{1}, x_{2}\right)\right):=k^{(0)} d x_{1} d x_{2}$ on the plane $\mathrm{R}^{2}$ is invariant with respect to the mapping $\phi_{1}: \mathbb{R}^{2} \rightarrow \mathbb{R}^{2}$. If to state now that this invariant measure is unique on the plane $\mathrm{R}^{2}$ this will mean [2-5] that the mapping $\phi_{1}: \mathbb{R}^{2} \rightarrow \mathbb{R}^{2}$ is ergodic. To show this; we will make use of the uniform discretization of the Frobenius-Perron operator (19) and find by means of usual numerical calculations that the corresponding $\mathrm{N}$-dimensional Frobenius-Perron matrix $\mathcal{P}_{\phi, N}: \mathbb{E}^{N} \rightarrow \mathbb{E}^{N}$ is irreducible for any dimension $N \rightarrow \infty$ This fact; owing to Proposition 2.2; makes it possible to formulate the following theorem.
Theorem 4.1: The Boole type transformation $\phi_{1}: \mathbb{R}^{2} \rightarrow \mathbb{R}^{2}$ of (18) is ergodic.

Concerning the mixing property of the mapping $\phi_{2}: \mathbb{R}^{2} \rightarrow \mathbb{R}^{2}$ additional calculations still are needed to show; owing to Proposition 2.3; that the $N$-dimensional Frobenius-Perron matrix $\mathcal{P}_{\phi, N}: \mathbb{E}^{N} \rightarrow \mathbb{E}^{N}$ is $q_{\mathrm{N}}$-maximal cycling for any dimension $N \rightarrow \infty$.

Remark 4.2: Taking into account that the mapping $\phi_{2}: \mathbb{R}^{2} \rightarrow \mathbb{R}^{2}$ is simply conjugated with the mapping $\phi_{1}: \mathbb{R}^{2} \rightarrow \mathbb{R}^{2}$ all statements above concerning its ergodicity also hold for the mapping $\phi_{2}: \mathbb{R}^{2} \rightarrow \mathbb{R}^{2}$

The Boole type mappings (18) can be generalized on the threedimensional space $\mathbb{R}^{3:}$

$$
\begin{aligned}
& \varphi_{1}\left(x_{1}, x_{2}, x_{3}\right):=\left(x_{1}-1 / x_{2}, x_{2}-1 / x_{3}, x_{3}-1 / x_{1}\right), \\
& \varphi_{2}\left(x_{1}, x_{2}, x_{3}\right):=\left(x_{1}-1 / x_{3}, x_{2}-1 / x_{1}, x_{3}-1 / x_{2}\right),
\end{aligned}
$$

defined for any $\left(x_{1}, x_{2}, x_{3}\right) \in \mathbb{R}^{3},\{(0,0,0)\}$ It was already proved in ref. [16] that these mapping are invariant with respect to the standard Lebesgue measure $d v\left(x_{1}, x_{2}, x_{3}\right)=d x_{1} d x_{2} d x_{3}$ on $\mathbb{R}^{3}$ yet their ergodicity is still under investigation.

\section{Acknowledgements}

The author would like to convey his cordial thanks to Prof. Denis Blackmore (NJIT; Newark NJ; USA) for instrumental discussion during the collaborative research stay at the NJIT; NJ USA during May 25-31; 2015; as well as for the related comments and useful remarks. He is specially acknowledged to $\mathrm{AGH}$ of Krakow (Poland) for partial supporting this research and a travel fund for the SIAM Conference on "Dynamical Systems" held in 20-25 May 2015.

\section{References}

1. Aaronson $J$ (1997) An introduction to infinite ergodic theory. American Mathematical Society.

2. Katok A, Hasselblatt B (1999) Introduction to the Modern Theory of Dynamical Systems. Cambridge Univ Press, UK.

3. Pollicott M, Yuri M (1998) Dynamical systtems and ergodic theory. Cambridge University Press, UK.

4. Walters $P$ (1975) An Introduction to Ergodic Theory. Springer; New York.

5. Einsiedler M, Ward T (2011) Ergodic theory: Graduate Texts in Mathematics. Springer, London.

6. Kreuzer E (1987) Numerische untersuchung nichlinearer dynamischer Systeme. Springer Berlin.

7. Li TY (1976) Finite approximation for the Frobenius-Perron operator. A solution to Ulam's conjecture. J Approx Theory 17: 177-186.

8. Hotzel W, Lehmkuhl N, Werner B (2002) The spectrum of the Frobenius-Perron operator and its dis cretization for circle diffeomorphisms. Journal of Dynamics and Differential Equations 14: 443-461.

9. Lasota A, Mackey C (1994) Chaos; fractal and noise: stochastic aspects of dynamics; Springer.

10. Keller $\mathrm{G}$ (1984) On the rate of covergence to equilibrium in one-dimensional systems. Comm Math Phys 96: 181-193.

11. Dellnitz M, Hohmann A, Junge O, Rumpf M (1997) Exploring invariant serts and invariant measures. Chaos Solitons and Fractals 7: 221-228.

12. Guder R, Dellnitz M, Kreuzer E (1997) An adaptive method for the approximation of the generalized cell mapping. Chaos; Solitons and Fractals 8: 525-534.

13. Berman A, Plemmons RJ (1979) Non-negative matrices in the mathematical scince. Academic Press London.

14. Boole G (1857) On the comparison of transcendents with certain applications to the theory of definite integrals. Phil Trans Roy Soc 147: 745-803.

15. Adler R, Weiss B (1973) The ergodic; infinite measure preserving transformation of Boole. Isr J Math 16: 263-278.

16. Blackmore D, Golenia J, Prykarpatsky AK, Prykarpatsky YA (2013) Infvariant meassures for discrete dynamical systems and ergodic properties of generalized Boole type transformations. Ukrainian Mathematical Journal 65: 47-63. 